The Astrophysical Journal, 655:L105-L108, 2007 February 1

(C) 2007. The American Astronomical Society. All rights reserved. Printed in U.S.A.

\title{
MOLECULAR GAS IN THE INNER 1 AU OF THE TW HYA AND GM AUR TRANSITIONAL DISKS
}

\author{
C. Salyk, ${ }^{1}$ G. A. Blake, ${ }^{1}$ A. C. A. Boogert, ${ }^{2}$ and J. M. Brown ${ }^{3}$ \\ Received 2006 June 6; accepted 2006 December 20; published 2007 January 16
}

\begin{abstract}
We report the detection of $\mathrm{CO}$ rovibrational emission from the transitional disks around the T Tauri stars TW Hya and GM Aur. Transitional disks are characterized by significant mid- to far-infrared (IR) dust emission combined with a relative deficit in the near-IR, indicating the presence of an optically thick outer disk but a reduced surface density of small dust grains in the inner disk. Kinematic fits to the resolved CO emission lines demonstrate that they arise from within the tenuous inner disk. Excitation diagram analyses yield rotational temperatures also consistent with small emission radii as well as densities implying dynamically significant amounts of gas in the inner disk and a gas-to-small dust grain ratio in excess of that in dense clouds. Nevertheless, gas densities are not high enough to maintain current accretion rates for more than a few hundred years without replenishment, and transfer of gas from the outer to inner disk is therefore likely required.
\end{abstract}

Subject headings: planetary systems: protoplanetary disks — stars: pre-main-sequence

\section{INTRODUCTION}

As the intersection between star and disk processes and the likely location of terrestrial planet formation, the inner regions of circumstellar disks $(R \lesssim 5 \mathrm{AU})$ hold information essential for understanding the evolution of young stars and planetary systems. In particular, inner disk gas controls such varied processes as planetary migration rates (Ward 1997), orbit circularization (Kominami \& Ida 2002), and accretion (Shu et al. 1994). However, because of their small size and proximity to the bright parent star, inner disks are notoriously difficult to image. Recently, rovibrational emission lines of species such as $\mathrm{H}_{2} \mathrm{O}$ (Carr et al. 2004) and CO have emerged as ideal probes of the near-surface layers of inner disks. Fundamental $\mathrm{CO}$ rovibrational emission $(v=1 \rightarrow 0)$ has been observed from embedded protostars (Pontoppidan et al. 2002), classical T Tauri stars (Najita et al. 2003; Rettig et al. 2004), and Herbig Ae/Be stars (Brittain et al. 2003; Blake \& Boogert 2004).

CO $v=1 \rightarrow 0$ emission also provides a unique view into the inner regions of so-called transitional disks, whose spectral energy distributions (SEDs) are characterized by nearly photospheric levels of emission in the near-IR but significant excess flux at mid- to far-IR wavelengths. They are thus believed to have optically thin inner disks or gaps caused by grain growth and/or clearing due to dynamical interactions (Lin \& Papaloizou 1979), perhaps due to the presence of a planet (Rice et al. 2003; Calvet et al. 2002). Two prototypical examples of transitional disks are TW Hya (K8 V) and GM Aur (K5 V), whose SEDs show evidence of significant disk clearing out to $\sim 4$ AU (Calvet et al. 2002) and 24 AU (Rice et al. 2003), respectively. For TW Hya, SED fits yield an inner disk dust mass of $\sim 6 \times 10^{-3} M_{\oplus}$ (Calvet et al. 2002), while a $K$-band interferometric study yields values closer to $\sim 5 \times 10^{-7} M_{\oplus}$ (Eisner et al. 2006). There is an estimated $2.5 \times 10^{-4} M_{\oplus}$ of dust encircling GM Aur, confined to within $\sim 5$ AU from the star (Calvet et al. 2005). Accretion rates for TW Hya and GM Aur are lower than that of average T Tauri stars: $\sim 4 \times 10^{-10}$

\footnotetext{
${ }^{1}$ Division of Geological and Planetary Sciences, California Institute of Technology, Pasadena, CA; csalyk@gps.caltech.edu.

${ }^{2}$ NOAO, Gemini Science Center, La Serena, Chile.

${ }^{3}$ Division of Physics, Mathematics and Astronomy, California Institute of Technology, Pasadena, CA.
}

$M_{\odot} \mathrm{yr}^{-1}$ (Muzerolle et al. 2000) and $\sim 10^{-8} M_{\odot} \mathrm{yr}^{-1}$ (White \& Ghez 2001).

Observations of $\mathrm{CO} v=1 \rightarrow 0$ emission from TW Hya were first reported by Rettig et al. (2004). We expand on these results, reporting NIRSPEC observations of $\mathrm{CO}$ emission from TW Hya that cover a larger range of rotational energies $\left(E_{j} \sim\right.$ $3000-6000 \mathrm{~K}$ vs. $\sim 3200-4700 \mathrm{~K})$ as well as Phoenix observations for which the emission lines are spectrally resolved. We also report the first observations of $\mathrm{CO} v=1 \rightarrow 0$ emission from GM Aur.

\section{OBSERVATIONS}

$M$-band spectra were obtained with NIRSPEC (McLean et al. 1998) at the Keck II telescope on 2005 April 24-25 and December 18, 20 for TW Hya and 2004 December 27, 30 for GM Aur. Echelle mode observations with the $0.43^{\prime \prime} \times 24^{\prime \prime}$ slit (yielding a Gaussian FWHM of $\sim 12.5 \mathrm{~km} \mathrm{~s}^{-1}$, as measured on unresolved $\mathrm{CO}$ absorption lines) encompassed the first two $R$ branch lines and many $P$-branch lines [specifically, $P(1 \rightarrow 12)$ and $P(30 \rightarrow 40)$ ] of the $v=1 \rightarrow 0 \mathrm{CO}$ rovibrational band. Further Phoenix (Hinkle et al. 2003) spectra of a few TW Hya lines were obtained at Gemini South on 2006 April 6-7 using the $0.25^{\prime \prime} \times 14^{\prime \prime}$ slit (measured FWHM $\sim 5 \mathrm{~km} \mathrm{~s}^{-1}$ ).

The two-dimensional echellograms were analyzed with the procedures outlined in Boogert et al. (2002) using standard stars (HR 1620 [A7 V], HR 4313 [A2 V], HR 4494 [B9 V], and HR 5028 [A2 V]) for atmospheric correction and flux calibration. After removal of wavelengths with significant telluric absorption (transmission $\$ 40 \%-65 \%$ ), flux-calibrated spectra with signal-to-noise ratios approaching the shot-noise limit can be obtained. For TW Hya, which was observed twice with Doppler shifts of $\sim 12$ and $\sim 24 \mathrm{~km} \mathrm{~s}^{-1}$, most line profiles remain complete after the removal of potential telluric interference. For GM Aur, observed only once with a Doppler shift of $\sim 11 \mathrm{~km} \mathrm{~s}^{-1}$, line profiles are incomplete. However, more than half of the profile remains, allowing reasonable constraints on both the height and the width of the lines. Although lineto-continuum variability by a factor of 10 or more for TW Hya has been reported (Rettig et al. 2004), we saw variations consistent with noise, with line-to-continuum ratios varying from 0.6 to 1 [for the $P(5)-P(7)$ lines].

TW Hya shows CO $v=1 \rightarrow 0 R(0-1), P(1-12)$, and $P(30-$ 32) lines. GM Aur was observed in one additional setting, and 
its spectrum shows emission out to at least $P(14)$. No ${ }^{13} \mathrm{CO}$ $v=1 \rightarrow 0$ or $\mathrm{CO} v=2 \rightarrow 1$ emission is seen. The line and continuum spatial FWHM of $\sim 0.5^{\prime \prime}$ are consistent with the $0.4^{\prime \prime}-$ $0.7^{\prime \prime} K$-band seeing, and they constrain the emission to radii $<10-15$ AU for TW Hya and <30-35 AU for GM Aur. Excerpts of our NIRSPEC $M$-band spectra are shown in Figure 1.

\section{RESULTS}

\subsection{Line Widths}

Because $\mathrm{CO} v=1 \rightarrow 0$ emission originates from the inner regions of Keplerian disks, line shapes depend on the disk inclination and the emitting radius (Najita et al. 2003; Blake \& Boogert 2004). For TW Hya's nearly face-on disk, the NIRSPEC data yield only marginally resolved lines, so we instead utilize the $R=60,000$ Phoenix spectra. A composite line shape from the $P(6)-P(9)$ transitions is presented as an insert in Figure 1. Individual lines were velocity-centered, continuum-subtracted, normalized, and resampled into $2 \mathrm{~km} \mathrm{~s}^{-1}$ bins.

Inner emission radii are often estimated from the half-width at zero intensity (HWZI). This measurement yields an inner radius for TW Hya of $\sim 0.04 \mathrm{AU}$ (for $M_{\star}=0.6 M_{\odot}$ and $i=$ $7^{\circ} \pm 1^{\circ}$; Qi et al. 2004) but does not take into account disk structure or any non-Keplerian (turbulent) motions, whose lineof-sight projections are especially important for low-inclination disks. A complete two-dimensional disk model could not be constrained with only the data shown here, and we therefore present a compromise - a disk model consisting of an optically thick dust layer and overlying hotter gas layer, each with $T=$ $T_{0}(r / \mathrm{AU})^{\alpha}\left(L / L_{\odot}\right)$. Based on SED fits using the radiative transfer models of Dullemond et al. (2002) and on matching total line fluxes, we chose the following model parameters: $T_{0}$ (dust) $=$ $3450 \mathrm{~K}, T_{0}$ (gas) $=4400 \mathrm{~K}, \alpha=-0.2$, and $r_{\text {out }}=2 \mathrm{AU}$. Stellar and disk parameters $-M, i$ as above, and $L=0.25 L_{\odot}$ (Thi et al. 2004) — are derived from other studies, and $v_{\text {turb }}$ is parameterized as $0.01 v_{\text {Kep }}$. Resultant line shapes were convolved with the known Phoenix instrument response function (IRF).

The best least-squares fit has $r_{\text {in }}=0.2 \mathrm{AU}$. Including all trials within the $\chi^{2} 95 \%$ confidence interval, where $\sigma$ is the flux variation past the line profile wings, inner radii consistent with the data are $r_{\text {in }}=0.2_{-0.05}^{+0.2}$. Note that this estimate is several times larger than that estimated from the HWZI. A complete characterization of disk parameters and their influence on $r_{\text {in }}$ is not possible with this data set; nevertheless, we tried to get a feel for the magnitude of the importance of a few key parameters.

For nearly face-on disks such as TW Hya, the turbulent velocity is the most influential parameter at fixed inclination. For example, with $v_{\text {turb }} \sim 0.05 v_{\text {Kep }}$, the best-fit radii are $\sim 1 \mathrm{AU}$, but at such distances the disk may be too cool to induce the observed line fluxes. Doppler broadening at the temperatures of interest is unimportant, but the temperature gradient can be significant. Trials with $\alpha=-0.44$ and -0.05 result in $r_{\text {in }}$ up to $0.4 \mathrm{AU}$, so true error bars should be larger than the $\chi^{2}$ estimate by a factor of a few. TW Hya's outer disk inclination is well constrained, so inclination errors are a relatively insignificant source of error, affecting $r_{\text {in }}$ by $\$ 40 \%$, unless the disk is strongly warped.

The CO emission lines from GM Aur have a FWHM of 30 $\mathrm{km} \mathrm{s}^{-1}$, and so the NIRSPEC IRF makes a minor contribution to the measured line profile. A composite line was created utilizing $P(9)-P(12)$ and $P(14)$ (see Fig. 1 insert), and a HWZI estimate yields an inner emission radius of $0.1 \mathrm{AU}$ (with $M=0.84 M_{\odot}, i=54^{\circ} \pm 5^{\circ}$; Simon et al. 2000). Utilizing our two-layer disk model with the same parameterization as for

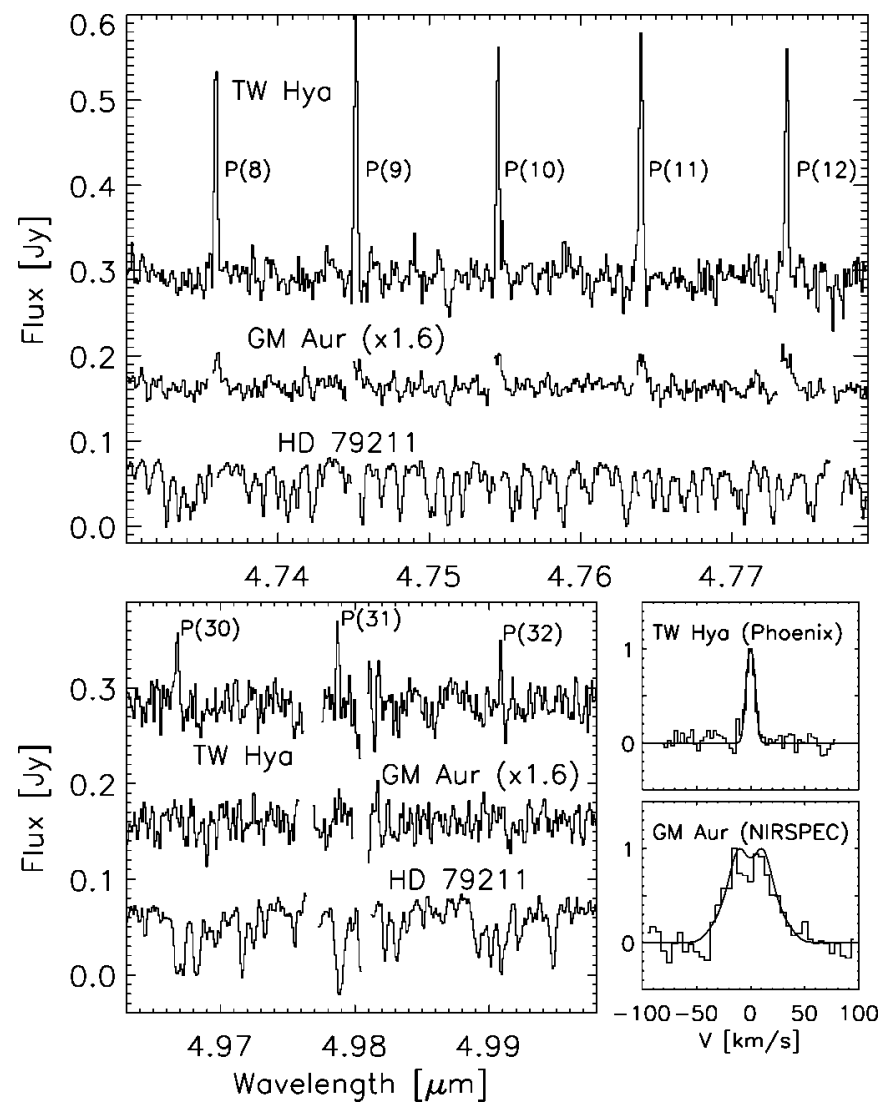

FIG. 1.-Excerpts of the NIRSPEC spectra of TW Hya and GM Aur (multiplied by 1.6). The HD 79211 spectrum has been normalized to TW Hya's continuum and offset. Line composites are shown at lower right, with the best two-layer disk model overlaid.

TW Hya, with stellar mass and disk inclination as above, and with $L=0.74 L_{\odot}, r_{\text {out }}=5 \mathrm{AU}$, we find that $r_{\text {in }}=0.3 \mathrm{AU}$. A $\chi^{2} 95 \%$ confidence interval metric yields $r_{\text {in }}=0.3_{-0.15}^{+0.2}$. Turbulence and inclination errors have little effect $(\leqslant 15 \%)$ on this high-inclination disk, but changes in $\alpha$ can still affect $r_{\text {in }}$ by factors of $\sim 2$.

\subsection{Excitation Diagrams}

To estimate gas temperatures and mass surface densities, we construct excitation diagrams, in which $\ln \left[F_{j i} /\left(g_{j} A_{j i} \nu_{j i}\right)\right]$ is plotted against $E_{j}$. Here, $F_{j i}$ is the line flux, $A_{j i}$ is the spontaneous emission coefficient, $\nu_{j i}$ is the wavenumber of the transition, $g_{j}(=2 j+1)$ is the upper state degeneracy, and $E_{j}$ is the upper state energy. For optically thin, single-temperature emission, the dependence is linear, with a slope of $-1 / T$, where $T$ is the excitation temperature that characterizes the rotational manifold, and an intercept that yields the column density. Nonlinear dependencies can indicate temperature gradients or optically thick emission.

Before calculating line fluxes, we first estimate the reduction in observed line fluxes induced by absorption features in the photospheres of TW Hya and GM Aur. Although the photospheres are nearly unobstructed at $1 \mu \mathrm{m}$ (Edwards et al. 2006), veiling appears to dominate in the $M$ band. We quantify this effect by fitting the spectra (away from the $v=1 \rightarrow 0$ CO lines) with the sum of a constant veiling term plus a scaled photospheric template, given by NIRSPEC data for the K7 V star HD 79211 (see Fig. 1). The veiling and median photospheric contributions are $0.197 \pm 0.006$ and $0.096 \pm 0.006 \mathrm{Jy}$ for 

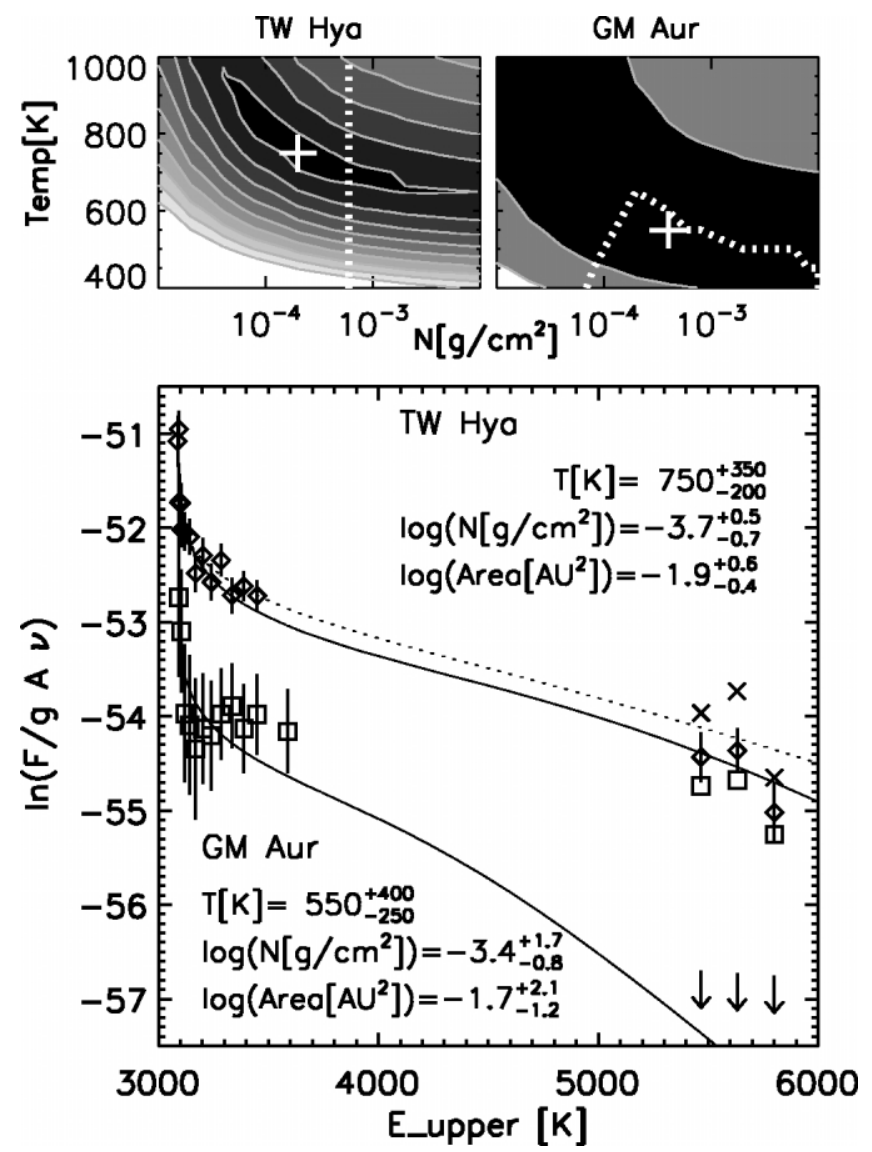

FIG. 2.-Excitation diagrams for TW Hya and GM Aur. Raw data are shown as squares, photospheric + veiling-corrected fluxes as diamonds, and veilingfree fluxes as crosses. Arrows denote upper limits, and best model fits are shown as solid lines (or dashed for veiling-free fluxes). We show $\chi^{2}$ contours for column density and temperature with area fixed at best-fit value. The innermost contour is a 95\% confidence interval. Dotted lines are additional constraints. Quoted limits are the fit parameter ranges consistent with the data.

TW Hya and $0.081 \pm 0.002$ and $0.019 \pm 0.002$ Jy for GM Aur, respectively. Here the error bars reflect only the statistical uncertainties of the fit. Subtraction of the photosphere + veiling model should now yield the "true" disk line fluxes. For TW Hya, $R$ and $P(1)-P(12)$ fluxes are changed by $\sim 6 \%$, on average, but $P(30)-P(32)$ fluxes are increased by $\sim 30 \%$. For GM Aur, the moderate signal-to-noise ratio and the dominance of veiling render flux corrections insignificant (photospheric fluctuations are of order $0.001 \mathrm{Jy}$, while the noise level is $\sim 0.01 \mathrm{Jy}$ ).

If HD 79211 is not a proper template, or if the photospheric absorption lines are filled in by hot gas from the inner disk or accretion flow, our fits will tend to underestimate the photospheric contribution. Therefore, to provide an upper limit to the possible photospheric impact, we considered the case in which the spectra between the CO $v=1 \rightarrow 0$ emission lines are assumed to be "veiling-free." Specifically, the model photosphere was scaled such that its median matched that of the source spectrum and was then subtracted from the data to derive veiling-free fluxes. TW Hya $R$ and $P(1)-P(12)$ line fluxes are changed by $7 \%$, on average, while $P(30)-P(32)$ fluxes are increased by up to $160 \%$. Again, GM Aur's line fluxes are not significantly affected because variations in the reference photosphere $(\sigma \sim 0.007 \mathrm{Jy})$ are smaller than the noise.

The excitation diagram in Figure 2 includes fluxes of 17 identifiable emission lines for TW Hya and 12 for GM Aur, as listed in Table 1. Nonlinearity is clearly present; therefore, the optically thin, isothermal assumption is invalid. To fully model the line emission, one would need to consider both the gas and dust distributions and temperatures as well as the disk geometry. However, to derive a simple intuitive estimate of conditions, we fit our excitation diagrams with a constant dust continuum plus a single-temperature gas whose column density, rotational temperature, and emitting area are free parameters. Gaussian line shapes derived from the data are also provided for the model.

The best fit to TW Hya's veiling + photosphere-corrected fluxes is described in Figure 2. Error bars are defined by the range of models constrained by a $95 \% \chi^{2}$ confidence interval and an upper density limit provided by the nondetection of ${ }^{13} \mathrm{CO}$.

TABLE 1

LINE FLuXES

\begin{tabular}{|c|c|c|c|c|c|c|c|c|}
\hline \multirow[b]{2}{*}{ LINE } & \multicolumn{6}{|c|}{ TW HYA } & \multicolumn{2}{|c|}{ GM AUR } \\
\hline & $F_{0}$ & FWHM & $F_{c}$ & FWHM & $F_{\mathrm{nv}}$ & FWHM & $F_{0}$ & FWHM \\
\hline$R(1) \ldots \ldots$ & $5.0 \pm 1.0$ & 14.5 & $5.1 \pm 1.3$ & 14.6 & $5.4 \pm 1.3$ & 15.0 & $1.3 \pm 0.9$ & 22.8 \\
\hline$R(0) \ldots \ldots$ & $5.5 \pm 1.1$ & 15.5 & $5.5 \pm 1.3$ & 15.1 & $5.6 \pm 1.4$ & 14.6 & $0.9 \pm 0.8$ & 15.6 \\
\hline$P(1) \ldots \ldots$ & $2.9 \pm 0.8$ & 16.8 & $4.8 \pm 1.1$ & 22.8 & $3.7 \pm 1.0$ & 15.0 & $\ldots$ & $\ldots$ \\
\hline$P(2) \ldots \ldots$ & $5.2 \pm 1.0$ & 20.7 & $5.0 \pm 1.1$ & 20.8 & $5.0 \pm 1.1$ & 22.2 & $\ldots$ & $\ldots$ \\
\hline$P(3) \ldots \ldots$ & $5.9 \pm 1.1$ & 21.0 & $5.5 \pm 1.2$ & 20.3 & $5.1 \pm 1.1$ & 19.5 & $\ldots$ & $\ldots$ \\
\hline$P(4) \ldots \ldots$ & $7.1 \pm 1.4$ & 13.5 & $7.2 \pm 1.8$ & 13.6 & $7.5 \pm 1.8$ & 13.9 & $1.0 \pm 0.8$ & 19.6 \\
\hline$P(5) \ldots \ldots$ & $8.3 \pm 1.6$ & 14.2 & $8.4 \pm 2.0$ & 14.3 & $8.5 \pm 2.0$ & 14.5 & $1.1 \pm 0.8$ & 18.0 \\
\hline$P(6) \ldots \ldots$ & $6.7 \pm 1.3$ & 13.8 & $6.8 \pm 1.7$ & 13.9 & $7.0 \pm 1.7$ & 14.0 & $1.1 \pm 0.8$ & 18.0 \\
\hline$P(7) \ldots \ldots$ & $9.5 \pm 1.7$ & 14.5 & $9.5 \pm 2.2$ & 14.7 & $9.8 \pm 2.2$ & 15.2 & $1.5 \pm 0.9$ & 26.9 \\
\hline$P(8) \ldots \ldots$ & $8.3 \pm 1.6$ & 13.6 & $8.1 \pm 2.0$ & 13.4 & $7.9 \pm 2.0$ & 13.1 & $1.6 \pm 0.9$ & 25.4 \\
\hline$P(9) \ldots \ldots$ & $11.3 \pm 1.9$ & 15.3 & $11.5 \pm 2.5$ & 15.5 & $11.9 \pm 2.5$ & 15.9 & $2.2 \pm 1.1$ & 37.2 \\
\hline$P(10) \ldots \ldots$ & $8.8 \pm 1.7$ & 13.8 & $8.7 \pm 2.1$ & 13.7 & $8.5 \pm 2.1$ & 13.4 & $2.7 \pm 1.2$ & 44.6 \\
\hline$P(11) \ldots \ldots$ & $10.6 \pm 1.7$ & 16.5 & $10.5 \pm 2.2$ & 16.2 & $10.3 \pm 2.2$ & 15.8 & $2.3 \pm 1.1$ & 35.6 \\
\hline$P(12) \ldots \ldots$ & $9.9 \pm 1.7$ & 16.0 & $10.2 \pm 2.1$ & 16.2 & $10.7 \pm 2.2$ & 16.6 & $2.9 \pm 1.2$ & 42.9 \\
\hline$P(14) \ldots \ldots$ & & & & & & & $2.8 \pm 1.2$ & 46.3 \\
\hline$P(30) \ldots \ldots$ & $2.9 \pm 0.8$ & 18.5 & $3.9 \pm 1.0$ & 21.4 & $6.3 \pm 1.2$ & 26.5 & $\ldots$ & $\ldots$ \\
\hline$P(31) \ldots \ldots$ & $3.1 \pm 0.7$ & 15.6 & $4.3 \pm 1.0$ & 18.2 & $8.1 \pm 1.3$ & 27.1 & $\ldots$ & $\ldots$ \\
\hline$P(32) \ldots \ldots$ & $1.8 \pm 0.6$ & 11.8 & $2.3 \pm 0.7$ & 12.6 & $3.3 \pm 0.9$ & 14.0 & $\ldots$ & $\ldots$ \\
\hline
\end{tabular}

Note. - Fluxes are in units of $10^{-18} \mathrm{~W} \mathrm{~m}^{-2}$. Error bars include two contributions added in quadrature: one from the standard deviation of the continuum flux and one from the standard deviation of fit line widths. $F_{0}$ is flux with no photospheric correction, $F_{c}$ is photospheric+veiling-adjusted flux, and $F_{\mathrm{nv}}$ is flux adjusted assuming no veiling. FWHM is in units of $\mathrm{km} \mathrm{s}^{-1}$. 
Note that area and $N$ tend to covary, and are both anticorrelated with $T$, such that fits with high $T$ correspond to low $N$ and area, and vice versa. The veiling-free fluxes (representing an upper limit) give only a slightly different temperature and area: $\log N=-3.7_{-0.4}^{+0.5} \mathrm{~g} \mathrm{~cm}^{-2}, T=950_{-300}^{+550} \mathrm{~K}$, and $\log ($ area $)=$ $-2.3_{-0.6}^{+0.6} \mathrm{AU}^{2}$. A lower temperature and density $(T=430 \pm$ $40 \mathrm{~K}, N \sim 1 \times 10^{-8} \mathrm{~g} \mathrm{~cm}^{-2}$ ) were derived by Rettig et al. (2004) assuming optically thin lines and an emitting area equal to that sampled by NIRSPEC (i.e., out to several tens of AU). However, given the curvature below $\sim 3300 \mathrm{~K}$ in Figure 2 and the high temperatures required to excite the transitions, these assumptions are not appropriate. For blackbodies, our fit temperatures correspond to $r=0.07_{-0.05}^{+0.36}$ and $0.04_{-0.03}^{+0.39} \mathrm{AU}$, but warm gas can exist at larger radii in disks with superheated atmospheres (e.g., Kamp \& Dullemond 2004).

Best fits for GM Aur can also be seen in Figure 2. In this case, the $\chi^{2}$ interval is significantly larger, and we used three additional limits to constrain our fits-a limit based on nondetection of emission at high $E_{j}$, a density limit imposed by nondetection of ${ }^{13} \mathrm{CO}$, and a requirement that fit models have significant curvature at low energies. The best-fit temperature corresponds to $r=0.53_{-0.16}^{+0.94} \mathrm{AU}$ for blackbodies.

\section{DISCUSSION}

The molecular emission we observe originates from inside 1 AU-well inside the estimated disk transition radii of $\sim 4$ and 24 AU for TW Hya and GM Aur, respectively. Line profiles yield inner radii of $0.2_{-0.05}^{+0.4}$ and $0.3_{-0.15}^{+0.2} \mathrm{AU}$, and rotational temperatures are consistent with emission radii of $r=0.07_{-0.05}^{+0.36}$ and $r=0.53_{-0.16}^{+0.94}$ AU for equilibrium blackbodies. These suggest CO-emitting layer temperatures similar to those for blackbodies in equilibrium with the stellar radiation.

Although the $\mathrm{CO}$ lies inside the optically thick transition radius derived from SEDs, it lies outside the estimated disk truncation and dust destruction radii. For example, Keck interferometry plus IR photometry yield an inner radius of $0.06 \pm 0.01 \mathrm{AU}$ for TW Hya's dust disk, which is consistent with the magnetic truncation radius (Eisner et al. 2006). GM Aur's inner disk dust is truncated at $0.221_{-0.085}^{+0.082} \mathrm{AU}$, consistent with the dust sublimation radius (Akeson et al. 2005). In both cases, our measurements put the $\mathrm{CO}$ inner radius outside that of the dust, likely because the $\mathrm{CO}$ must be shielded from photodissociation; at our derived inner radii, radial vacuum-UV $(\lambda \sim 0.1 \mu \mathrm{m})$ optical depths are $>5-10$, according to dust $\left[\kappa=10^{3}\left(\nu / \nu_{k}\right) \mathrm{cm}^{2} \mathrm{~g}^{-1}\right]$ and disk mass surface density $\left[\Sigma=\Sigma_{0}(R / \mathrm{AU})^{-3 / 2}\right]$ models analogous to those in Eisner et al. (2006).

Column densities derived from fits to the excitation diagrams allow us to estimate the gas content in the inner disk and the gas-to-dust ratio. For TW Hya, the best fit yields a gas surface density of $\Sigma_{\text {gas }} \sim 1 \mathrm{~g} \mathrm{~cm}^{-2}$ for an $\mathrm{H}_{2} / \mathrm{CO}$ ratio of $5 \times 10^{3}$ (Lacy et al. 1994). (This molecular ratio, observed toward massive protostars, is among the lowest observed in the dense interstellar medium and thus should provide a reasonable lower limit to the total gas mass.) A comparison with dust column densities derived by Eisner et al. (2006) for TW Hya implies a gas-to-dust ratio greater than $10^{5}$ at $\sim 0.2 \mathrm{AU}$; a comparison with the much higher dust surface density derived from Calvet et al. (2002) implies a gas-to-dust ratio of 250. If we extrapolate our densities to $4 \mathrm{AU}$ with a surface density that scales as $r^{-1.5}$, we predict a total inner disk gas mass of $4 \times 10^{26} \mathrm{~g}$ $\left(0.07 M_{\oplus}\right)$.

The best fit to GM Aur's CO emission yields a gas surface density of $\Sigma_{\text {gas }} \sim 2 \mathrm{~g} \mathrm{~cm}^{-2}$. Extrapolating to $5 \mathrm{AU}$ yields a total inner disk gas mass of $2 \times 10^{27} \mathrm{~g}\left(0.3 M_{\oplus}\right)$. Given the total dust mass from Calvet et al. (2005), this implies a gas-to-dust ratio of $\sim 1000$.

For both stars, the inner disk mass could only sustain the observed accretion rate for a few hundred years without invoking transfer of material from the outer disk. Steady mass transfer across gaps may be caused by an embedded planet (Rice et al. 2003). Interestingly, the derived gas surface densities also approximate those shown to provide significant circularization of the orbits of planetary embryos in the terrestrial planet-forming region (of order $0.1-1 \mathrm{~g} \mathrm{~cm}^{-2}$ at $1 \mathrm{AU}$; Kominami \& Ida 2002) and therefore may play an important role in shaping the dynamics of any forming planetesimals.

The authors thank Ken Hinkle for his assistance with the Phoenix observations. We also acknowledge support from the NASA Spitzer and Origins programs. Data presented herein were obtained at the W. M. Keck Observatory, which is operated as a scientific partnership among the California Institute of Technology, the University of California, and NASA. The Observatory was made possible by the generous financial support of the W. M. Keck Foundation. This work also utilized the Gemini Observatory as part of program GS-2006A-C-10. Gemini is operated by the Association of Universities for Research in Astronomy, Inc., under a cooperative agreement with the NSF on behalf of the Gemini partnership.

\section{REFERENCES}

Akeson, R. L., et al. 2005, ApJ, 635, 1173

Blake, G. A., \& Boogert, A. C. A. 2004, ApJ, 606, L73

Boogert, A. C. A., Blake, G. A., \& Tielens, A. G. G. M. 2002, ApJ, 577, 271

Brittain, S. D., Rettig, T. W., Simon, T., Kulesa, C., DiSanti, M. A., \& Dello Russo, N. 2003, ApJ, 588, 535

Calvet, N., D’Alessio, P., Hartmann, L., Wilner, D., Walsh, A., \& Sitko, M. 2002, ApJ, 568, 1008

Calvet, N., et al. 2005, ApJ, 630, L185

Carr, J. S., Tokunaga, A. T., \& Najita, J. 2004, ApJ, 603, 213

Dullemond, C.P., van Zadelhoff, G.-J., \& Natta, A. 2002, A\&A, 389, 464

Edwards, S., Fischer, W., Hillenbrand, L., \& Kwan, J. 2006, ApJ, 646, 319

Eisner, J. A., Chiang, E. I., \& Hillenbrand, L. A. 2006, ApJ, 637, L133

Hinkle, K. H., et al. 2003, Proc. SPIE, 4834, 353

Kamp, I., \& Dullemond, C. P. 2004, ApJ, 615, 991

Kominami, J., \& Ida, S. 2002, Icarus, 157, 43

Lacy, J. H., Knacke, R., Geballe, T. R., \& Tokunaga, A. T. 1994, ApJ, 428, L69

Lin, D. N. C., \& Papaloizou, J. 1979, MNRAS, 188, 191
McLean, I. S., et al. 1998, Proc. SPIE, 3354, 566

Muzerolle, J., Calvet, N., Briceño, C., Hartmann, L., \& Hillenbrand, L. 2000, ApJ, 535, L47

Najita, J., Carr, J. S., \& Mathieu, R. D. 2003, ApJ, 589, 931

Pontoppidan, K. M., Schöier, F. L., van Dishoeck, E. F., \& Dartois, E. 2002, A\&A, 393, 585

Qi, C., et al. 2004, ApJ, 616, L11

Rettig, T. W., Haywood, J., Simon, T., Brittain, S. D., \& Gibb, E. 2004, ApJ, 616, L163

Rice, W. K. M., Wood, K., Armitage, P. J., Whitney, B. A., \& Bjorkman, J. E. 2003, MNRAS, 342, 79

Shu, F., Najita, J., Ostriker, E., Wilkin, F., Ruden, S., \& Lizano, S. 1994, ApJ, 429, 781

Simon, M., Dutrey, A., \& Guilloteau, S. 2000, ApJ, 545, 1034

Thi, W.-F., van Zadelhoff, G.-J., \& van Dishoeck, E. F. 2004, A\&A, 425, 955

Ward, W. R. 1997, Icarus, 126, 261

White, R. J., \& Ghez, A. M. 2001, ApJ, 556, 265 